\title{
Evaluación de las creencias actitudinales hacia la violencia en centros educativos: el CAHV-25
}

\author{
José A. Ruiz, Laura Llor, Tomás Puebla y Bartolomé Llor \\ Universidad de Murcia (España)
}

\begin{abstract}
El objetivo del presente trabajo es la obtención de un instrumento breve y manejable para la evaluación de las actitudes hacia la violencia escolar. Un banco inicial de 45 ítems, distribuidos en seis factores teóricos, construidos y revisados siguiendo criterios precisos, se administró a una muestra de 275 sujetos. Los análisis realizados permitieron depurar el cuestionario que finalmente constó de 25 ítems con cuatro factores: "Violencia como forma de diversión" (7 ítems); "Violencia para mejorar la autoestima" (5 ítems); "Violencia para manejar los problemas y las relaciones sociales" (6 ítems); y "Violencia percibida como legítima" (7 ítems). Tanto el cuestionario general, como los cuatro factores, mostraron alta consistencia interna (entre ,68 y ,90). El instrumento resultante es de fácil uso e interpretación y puede contribuir al desarrollo de modelos predictivos del comportamiento violento en contextos educativos.
\end{abstract}

Palabras clave: Actitudes, violencia escolar, adolescentes, construcción, escala.

Assessment of attitudinal beliefs towards violence in educational centres: el CAHV-25. A new questionnaire has been developed in order to have a brief and manageable instrument for the assessment of attitudes towards violence in educational centres. The original pool of 45 items with six theoretical factors, constructed and reviewed using accurate standards, was filled out by 275 subjects. The analyses concluded with the selection of 25 items with four factors: "Violence as a form of diversion" (7 items); "Violence to improve the self-esteem" (5 items); "Violence to manage socials problems and relationships" (6 items), and "Violence perceived as legitimate" (7 items). Both the questionnaire and all 4 factors show a high internal consistency (between .68 and .90). This instrument has got an easy use and interpretation and could be useful to develop predictive models of the violence behaviour in educational contexts.

Key words: Attitudes, school violence, adolescents, construction, scale.

Correspondencia: José Antonio Ruiz Hernández. Departamento de Psiquiatría y Psicología Social. Facultad de Psicología. Universidad de Murcia. Campus de Espinardo, C.P. 30100. Murcia (España).E-mail: jaruiz@um.es 
La violencia escolar se ha convertido en una fuente de investigación, relativamente reciente, destacando entre los primeros estudios de violencia entre iguales, los realizados por Heinemman (1972) y Olweus $(1973,1978)$. Actualmente es uno de los principales problemas escolares en los países desarrollados. Según García (2001) en el ranking de problemas a resolver en Estados Unidos, la violencia ocupaba el segundo lugar, tras el de las drogas, y en el Reino Unido, casi un 33\% de los chicos de 14 y 15 años llevan algún tipo de arma. Según Ortega y Mora-Merchán (2000), España está por debajo en índices de violencia en comparación con Italia y Alemania, en la misma línea que otros autores (Gázquez, Cangas, Pérez-Fuentes, Padilla y Cano, 2007), y en una situación similar respecto a Gran Bretaña e Irlanda. En España encontramos entre un $10 \%$ y un $11,4 \%$ de agresores y entre un 5\% y 5,4\% de víctimas (Cerezo y Esteban, 1994; Ortega, 1994). El Informe Nacional del Defensor del Pueblo (2007) realizado sobre una muestra de 3.000 alumnos de educación secundaria de todas las comunidades autónomas señala que un $4,8 \%$ de alumnos afirman haber sufrido una agresión física directa, siendo el insulto la forma de agresión más frecuente (38,5\% de alumnos que se declaran víctimas). Cangas, Gázquez, Pérez-Fuentes, Padilla y Miras (2007), en un estudio realizado en alumnos de secundaria de cuatro países europeos, encuentran que casi la mitad del alumnado ha visto o participado en conductas de intimidación, que tienen que ver con insultos, malas palabras en clase o peleas.

La conducta agresiva entre iguales, parece iniciarse en la escuela infantil e ir aumentando durante la infancia media, con un punto álgido de incidencia, entre los 9 y los 14 años, para descender a lo largo de la adolescencia (Del Barrio, Martín, Almeida y Barrios, 2003). La adolescencia supone un periodo de alto riesgo para las conductas desviadas, incluyendo la violencia, atribuyéndose este riesgo a los importantes cambios sociales y físicos de este período (Farrel y Sullivan, 2004; Lowry, Sleet, Duncan, Powell y Kolbe, 1995).

Aunque tradicionalmente, se ha asociado la agresividad humana con el género masculino, estudios recientes hablan, de que la diferenciación de género estaría relacionada con la intensidad del comportamiento agresivo. Generalmente, los chicos muestran conductas problemáticas más serias (Hawkins, Catalana y Millar, 1992; Kazdin, 1988; Word, Cowan y Baker, 2002) y mayor agresividad física o "manifiesta" (incluso desde los 2 ó 3 años de vida), mientras que las chicas, preferirían utilizar estrategias agresivas "no físicas", tales como: la "agresión indirecta" o "relacional" (Andréu, Peña y Graña, 2001; Björkvist, 1994; Eagly, 1987; Farrel y Sullivan, 2004). Así por ejemplo, Etchebarría, Apodaca, Eceiza, Fuentes y Ortiz, 2003) encontraron que los chicos se mostraban más agresivos con sus iguales y menos cooperativos con el profesorado, y sugieren que el control inhibitorio aumenta en chicas y desciende en chicos, lo que podría relacionarse con las diferencias en la conducta agresiva. 
Las investigaciones realizadas, han permitido identificar una serie de factores relacionados con la violencia escolar: factores sociales (conductas agresivas en los medios de comunicación, nivel sociocultural y económico bajo, o estrés social provocado por el desempleo, aislamiento social y factores interculturales), factores familiares (desestructuración de la familia, malos tratos y modelado violento, falta de negociación y diálogo, métodos de crianza excesivamente restrictivos o excesivamente laxos o falta de afecto entre los cónyuges) y factores personales (baja motivación para la escuela, conducta antisocial, impulsividad, pertenecer al género masculino o encontrarse en la etapa evolutiva de la adolescencia) (Ando, 2005; Beyers, Loeber, Wikström y Stouthamer-Loeber, 2001; Costa y Morales, 1998; Gázquez et al., 2007; Gázquez, Pérez, Lucas y Palenzuela, 2008; Gómez, Navarro y Ruiz, 2005b; Hoglund, 2007; Parault, 2007; Rodríguez y Paíno, 1994; Rutter, Giller y Hagell, 1998; Scandroglio et al., 2002; Tolan, 2006).

A nuestro entender, las actitudes hacia la violencia escolar, podrían ser particularmente relevantes, en relación a la conducta interpersonal agresiva en el contexto educativo (Gómez, Navarro y Ruiz, 2005a). Se ha propuesto, que mayor parte de las conductas se producen de forma espontánea y que las actitudes, las guían, en gran medida procesos psicológicos automáticos (Fazio, 1986; Krauss, 1995; Schuette y Fazio, 1995). Según esto, la relación entre actitud y conducta es máxima, cuando los sujetos no utilizan procesos deliberativos a la hora de realizar su comportamiento. Si tenemos en cuenta que las conductas agresivas de los adolescentes en el contexto educativo, tienen una vertiente más afectiva o emocional que instrumental, y por tanto tienden a ser impulsivas y no premeditadas, es posible concluir que la evaluación de las actitudes hacia la conducta violenta podría contribuir a los modelos predictivos de dicho comportamiento en el contexto educativo. En anteriores trabajos, habíamos desarrollado una escala breve de 8 ítems para evaluar las actitudes hacia la violencia escolar, con buenas propiedades psicométricas (Gómez et al., 2005a). El objetivo del presente trabajo, es la mejora del CAHV-8 mediante el desarrollo de un cuestionario más completo, para la evaluación de las actitudes hacia la violencia en adolescentes.

\section{METODO}

\section{Participantes}

Se utilizó una muestra de 275 de adolescentes procedentes de cuatro centros de educación secundaria de la Región de Murcia. Tras eliminar 6 cuestionarios con problemas en la respuesta, 269 sujetos formaron la muestra definitiva $(63,2 \%$ eran chicas y 36,8\% chicos). La edad media fue de 15,75 años y la desviación típica de 1,49, con un rango entre 12 y 18 años. La participación fue voluntaria, manteniendo una estricta confidencialidad en el tratamiento y uso de los datos obtenidos. 


\section{Instrumentos y procedimiento}

En primer lugar, se generó un conjunto de enunciados relacionados con creencias actitudinales sobre la violencia escolar, utilizando la técnica de brainstorming, en seis grupos de discusión, instruidos para la tarea y formados, por entre cuatro y seis colaboradores cada uno. En este proceso, se tuvo en cuenta la estructura de tres dimensiones de las actitudes: cognitiva, afectiva y conductual (Eagly y Chaiken, 1998); y se trató de evitar el sesgo de inercia en las respuestas, redactando también ítems que implicaban teóricamente puntuaciones inversas.

Se obtuvieron inicialmente 85 ítems, que fueron revisados y discutidos por expertos, eliminándose aquellos que tenían problemas de formulación y ajustando la redacción a los criterios de Edward (1957). Así, se obtuvieron un total de 45 ítems distribuidos según 6 factores teóricos: "Violencia como forma de diversión" (11 ítems); "Violencia para mejorar la autoestima" (11 ítems); "Violencia como forma de relacionarse socialmente" (5 ítems); "Violencia para afrontar problemas" (5 ítems); "Violencia para impartir justicia" (5 ítems); y "Violencia para defenderse" (8 ítems).

Los ítems fueron redactados para su respuesta mediante una escala de tipo Likert de 5 niveles, que indicaba el grado de acuerdo/desacuerdo con el enunciado del ítem (desde 1= muy en desacuerdo, a 5= muy de acuerdo).

El cuestionario incluyó, además, preguntas sobre aspectos sociodemográficos (edad, género, curso y nivel educativo). Los cuestionarios se aplicaron de forma anónima, por psicopedagogos en formación, en las aulas de los centros educativos.

Para estudiar la validez de las dimensiones propuestas y depurar la escala, se estudió la fiabilidad de las distintas dimensiones teóricas, propuestas mediante el alfa de Cronbach y análisis factoriales de componentes principales con rotación varimax, siguiendo el criterio descrito por Kaiser (1960). Para la reducción del número de ítems y depuración de la escala, se combinaron varios criterios: a) el autovalor del factor resultante debía ser superior a 1; b) cada factor debía explicar al menos el 5\% de la varianza total; c) la carga factorial de los ítems debía ser elevada; y d) los ítems contenidos en las dimensiones totales debían presentar una consistencia interna adecuada.

\section{RESULTADOS}

Partiendo de los 45 ítems iniciales que tenían una consistencia interna de 0,91, y siguiendo el procedimiento estadístico mencionado, se analizaron los 6 factores teóricos propuestos, para comprobar si tenían un correlato empírico. Tras los análisis realizados, la escala se redujo a 25 ítems, con un alfa de 0,90 y cuatro factores con una consistencia interna adecuada (ver tabla 1 y anexo). 
Tabla 1. Datos descriptivos y análisis de fiabilidad de la escala resultante: coeficiente de fiabilidad ( $\alpha$ de Cronbach) y correlación ítem-factor

\begin{tabular}{|c|c|c|c|c|c|}
\hline Factor & Ítem & Media & $\begin{array}{l}\text { Desv. } \\
\text { Tip. }\end{array}$ & $\begin{array}{l}\text { Corr. } \\
\text { Item- } \\
\text { Factor }\end{array}$ & $\begin{array}{l}\text { Coef. } \\
\text { Alfa }\end{array}$ \\
\hline \multirow{7}{*}{$\begin{array}{l}\text { I. Violencia } \\
\text { como forma de } \\
\text { diversión }\end{array}$} & $\begin{array}{l}\text { 45. Me resulta divertido meterme con algunos } \\
\text { compañeros }\end{array}$ & 2,11 & 1,11 & 0,56 & \multirow{7}{*}{0,78} \\
\hline & 09. Me gusta ver peleas en la escuela & 2,25 & 1,22 & 0,55 & \\
\hline & 42. Me gusta hablar de peleas con mis amigos & 2,18 & 1,14 & 0,54 & \\
\hline & 04. Me divierte reírme de algunos compañeros & 2,31 & 1,09 & 0,53 & \\
\hline & 16. Me gusta poner motes a mis compañeros & 2,65 & 1,15 & 0,50 & \\
\hline & 24. Meterse con los profesores es divertido & 2,55 & 1,23 & 0,49 & \\
\hline & 15. Pelearse puede ser una forma de divertirse & 1,71 & 1,11 & 0,40 & \\
\hline \multirow{5}{*}{$\begin{array}{l}\text { II. Violencia } \\
\text { para mejorar la } \\
\text { autoestima }\end{array}$} & 46. Me siento "fuerte" si me meto con mis compañeros & 1,55 & 0,79 & 0,65 & \multirow{5}{*}{0,78} \\
\hline & $\begin{array}{l}\text { 40. Me siento "fuerte" después de insultar a un } \\
\text { compañero }\end{array}$ & 1,60 & 0,83 & 0,58 & \\
\hline & $\begin{array}{l}\text { 48. Me siento bien agrediendo a los compañeros de } \\
\text { clase que me caen mal }\end{array}$ & 1,59 & 0,80 & 0,58 & \\
\hline & $\begin{array}{l}\text { 43. Me siento importante cuando demuestro mi fuerza a } \\
\text { los compañeros }\end{array}$ & 1,85 & 1,09 & 0,57 & \\
\hline & 38. Me gusta que los demás tengan miedo & 1,66 & 0,93 & 0,45 & \\
\hline \multirow{6}{*}{$\begin{array}{l}\text { III. Violencia } \\
\text { para manejar } \\
\text { los problemas y } \\
\text { las relaciones } \\
\text { sociales }\end{array}$} & $\begin{array}{l}\text { 32. A veces me meto con mis compañeros hasta } \\
\text { conseguir lo que quiero de ellos }\end{array}$ & 1,68 & 1,00 & 0,51 & \multirow{6}{*}{0,68} \\
\hline & $\begin{array}{l}\text { 06. En la escuela suelo resolver mis problemas usando } \\
\text { la fuerza }\end{array}$ & 1,65 & 0,92 & 0,45 & \\
\hline & 19. Hay cosas que hay que resolver por la fuerza & 2,35 & 1,24 & 0,42 & \\
\hline & 30. Controlo a mis amigos por medio de la amenaza & 1,50 & 0,81 & 0,41 & \\
\hline & $\begin{array}{l}\text { 36. Me gusta meterme con los demás para que se fijen } \\
\text { en mí }\end{array}$ & 1,52 & 0,86 & 0,37 & \\
\hline & 23. Pegando los demás te respetan & 2,37 & 1,28 & 0,35 & \\
\hline \multirow{7}{*}{$\begin{array}{l}\text { IV. Violencia } \\
\text { percibida como } \\
\text { legítima }\end{array}$} & 21. Me gustaría pegar a los que me insultan & 2,92 & 1,18 & 0,54 & \multirow{7}{*}{0,72} \\
\hline & $\begin{array}{l}\text { 14. Si me pegara un compañero no le devolvería el } \\
\text { golpe }(*)\end{array}$ & 3,74 & 1,19 & 0,45 & \\
\hline & 33. Pelearía para ayudar a un amigo & 3,90 & 1,01 & 0,44 & \\
\hline & $\begin{array}{l}\text { 41. Si me insultara un compañero me defendería } \\
\text { atacándole }\end{array}$ & 2,44 & 1,21 & 0,43 & \\
\hline & $\begin{array}{l}\text { 07. Me parece bien meterme con un compañero curando } \\
\text { se lo ha ganado }\end{array}$ & 2,82 & 1,24 & 0,42 & \\
\hline & $\begin{array}{l}\text { 44. Me sentiría mal conmigo mismo si para defender a } \\
\text { un amigo tengo que pegar a orto chico }(*)\end{array}$ & 3,06 & 1,36 & 0,41 & \\
\hline & 34. La violencia es adecuada para defenderse & 2,66 & 1,30 & 0,36 & \\
\hline \multicolumn{2}{|l|}{ (*) Ítems inversos } & \multicolumn{3}{|c|}{ ALFA TOTAL } & 0,90 \\
\hline
\end{tabular}

El primer factor denominado teóricamente, "Violencia como forma de diversión", partía de 11 ítems que tenían un $\alpha=0,75$. Tras realizar un análisis factorial y estudiar la matriz de componentes rotados, se seleccionaron los seis ítems que cargaban en el componente principal con pesos factoriales entre 0,55 y 0,71. Estos 6 ítems, eran relativos a la violencia como medio de diversión o pasatiempo ("Me resulta divertido meterme con algunos compañeros"; "Me gusta ver peleas en la escuela",...). La subescala resultante mostró una consistencia interna de 0,78 , oscilando la correlación ítem-factor entre 0,40 y 0,56 .

El segundo factor que se denominó, "Violencia para mejorar la autoestima", partía de 11 ítems con un alfa de Cronbach de 0,78. Se seleccionó el primer componente del análisis factorial con los ítems que cargaban entre 0,55 y 0,77. El 
factor quedó constituido por 5 ítems que se refieren a una sensación de bienestar y mayor valía personal al utilizar la violencia ("Me siento "fuerte" si me meto con mis compañeros"; "Me siento "fuerte" después de insultar a un compañero",...). La consistencia interna de esta subescala fue de 0,78 .

Dos de los factores teóricos (falta de habilidades y resolución de conflictos) que tenían 5 ítems cada uno, mostraron consistencias internas muy bajas $(0,54$ y 0,40 , respectivamente). Dado que los contenidos de estos factores suelen ser categorizados desde la psicología, como déficits en la resolución de problemas, consideramos la posibilidad de estudiar los ítems como pertenecientes a un único factor que llamaríamos "Violencia para manejar los problemas y las relaciones sociales". La consistencia interna inicial de este factor resultó ser baja $(0,59)$, pero la depuración del factor permitió elevarla a 0,68. Los seis ítems seleccionados, se refieren al uso de la violencia en la resolución de problemas y el afrontamiento de situaciones sociales ("A veces me meto con mis compañeros hasta conseguir lo que quiero de ellos"; "En la escuela suelo resolver mis problemas usando la fuerza"; "Controlo a mis amigos por medio de la amenaza",...). La correlación ítem-factor fue de entre 0,35 y 0,51.

Finalmente, el último factor resultante de los análisis fue denominado "Violencia percibida como legítima" ya que contiene ítems procedentes de los dos últimos factores teóricos (la violencia justa y la violencia para defenderse). Ambos factores no mostraron una consistencia interna satisfactoria de forma separada pero, tras aplicar un análisis factorial y seleccionar los ítems del principal componente rotado, el $\alpha$ de Cronbach obtenido fue de 0,72 con 7 ítems ("Me gustaría pegar a los que me insultan"; "Pelearía para ayudar a un amigo"; "La violencia es adecuada para defenderse",...). Los ítems mostraron una correlación con la escala total que osciló entre 0,36 y 0,54 .

Tabla 2. Estudio de las relaciones entre variables y sus factores

\begin{tabular}{|c|c|c|c|c|c|c|c|c|c|c|c|}
\hline & & \multicolumn{2}{|c|}{ CAHV-25 } & \multicolumn{2}{|c|}{ FI } & \multicolumn{2}{|c|}{ FII } & \multicolumn{2}{|c|}{ FIII } & \multicolumn{2}{|c|}{ FIV } \\
\hline & & $\begin{array}{c}\text { Media } \\
\text { (DT) }\end{array}$ & Prob. & $\begin{array}{c}\text { Media } \\
\text { (DT) }\end{array}$ & Prob. & $\begin{array}{c}\text { Media } \\
\text { (DT) }\end{array}$ & Prob. & $\begin{array}{c}\text { Media } \\
\text { (DT) }\end{array}$ & Prob. & $\begin{array}{c}\text { Media } \\
\text { (DT) }\end{array}$ & Prob. \\
\hline \multirow[t]{4}{*}{ Género } & Chico & 2,54 & \multirow{4}{*}{$(* * *)$} & 2,53 & \multirow{4}{*}{$(* * *)$} & 1,87 & \multirow{4}{*}{$(* * *)$} & 2,15 & & 3,37 & \multirow{4}{*}{$(* * *)$} \\
\hline & $n=99$ & $(0,57)$ & & $(0,76)$ & & $(0,67)$ & & $(0,65)$ & $(* * *)$ & $(0,71)$ & \\
\hline & Chica & 2,10 & & 2,09 & & 1,52 & & 1,66 & (3) & 2,91 & \\
\hline & $n=170$ & $(0,54)$ & & $(0,72)$ & & $(0,61)$ & & $(0,57)$ & & $(0,72)$ & \\
\hline \multirow[t]{4}{*}{ Ciclo } & $E S O$ & 2,29 & \multirow{4}{*}{ n.s. } & 2,22 & \multirow{4}{*}{ n.s. } & 1,67 & \multirow{4}{*}{ n.s. } & 1,94 & & 3,12 & \multirow{4}{*}{ n.s. } \\
\hline & $n=130$ & $(0,60)$ & & $(0,76)$ & & $(0,61)$ & & $(0,66)$ & $(*)$ & $(0,78)$ & \\
\hline & Bachill. & 2,25 & & 2,28 & & 1,64 & & 1,75 & (") & 3,05 & \\
\hline & $n=139$ & $(0,58)$ & & $(0,79)$ & & $(0,69)$ & & $(0,62)$ & & $(0,72)$ & \\
\hline
\end{tabular}

$(* * *)=\mathrm{p}<0,001 ;(*)=\mathrm{p}<0,05 ;$ n.s. $=\mathrm{p}>0,05$

Estudiando las puntuaciones medias, comprobamos que la media más elevada $(M=3,08)$ se corresponde con el factor IV ("Violencia percibida como 
legítima") y la más baja $(\mathrm{M}=1,65)$ pertenece al factor II ("Violencia para aumentar la autoestima").

Al comparar las puntuaciones según el género, se observa, la presencia de puntuaciones estadísticamente mayores en chicos que en chicas, tanto en la escala general como en los factores de la escala (ver tabla 2). En cuanto al ciclo de estudio, únicamente aparecieron diferencias significativas en el caso del factor III, donde los alumnos pertenecientes a ESO puntuaban de forma significativamente mayor que los alumnos de Bachillerato ( $\mathrm{M}=1,94$ vs. $\mathrm{M}=1,75, \mathrm{t}=2,42, \mathrm{p}<0,05)$.

\section{DISCUSION}

En el presente estudio, hemos desarrollado un instrumento para evaluar las creencias actitudinales hacia la violencia escolar, en una muestra no clínica de adolescentes. Después de diversos análisis, el cuestionario resultante consta de 25 ítems y presenta una elevada consistencia interna. El estudio estadístico de los factores teóricos propuestos ha permitido confirmar la consistencia interna de cuatro factores: a) diversión; b) autoestima o aumento del bienestar; c) resolución de problemas sociales; y d) defensa.

Como principales ventajas de este instrumento destacan: 1) La alta consistencia interna, tanto del cuestionario completo, como de sus factores; 2) Debido a su formato, el fácil manejo y escaso tiempo que requiere su aplicación; 3) Fácil interpretación general y de los factores del cuestionario; 4) Gracias a las subescalas, nos permite estudiar con mayor detalle, la complejidad de las actitudes hacia la violencia. Estas características hacen que el CAHV-25 suponga una mejora considerable sobre el CAHV-8.

Consideramos, que los factores propuestos se relacionan con diversas líneas de investigación sobre el comportamiento violento. Por ejemplo, los factores de diversión y de defensa pueden relacionarse con el trabajo clásico de Beynon (1989) que sugería, que la violencia en los centros escolares está sometida a reglas sociales que regulan su uso y valoración, denominando "violencia divertida" a un tipo de violencia que gozaba del favor de los alumnos, formando parte de sus anécdotas preferidas, y "violencia justa" a aquella que era utilizada de forma adecuada o correcta.

Por otro lado, la "violencia para mejorar la autoestima", daría cuenta de las actitudes hacia el uso instrumental de la violencia, destinada a sentirse mejor con uno mismo. Diversos trabajos han mostrado, que el uso de la violencia, desprecio o prejuicios hacia los otros tiene efectos sobre el mantenimiento de la propia imagen, del bienestar o de la autoestima (Fein y Spencer, 1997; Taylor, Davis-Kean y Malanchuk, 2007). El recurso a la violencia para sentirse mejor consigo mismo sería mayor cuando no se dispone de otros medios para superar las amenazas a la autoimagen. 
Respecto a la subescala relacionada con el uso de la violencia para compensar los déficits en resolución de problemas y en habilidades sociales, consideramos que requiere de un mayor desarrollo, ya que el ó obtenido, está ligeramente por debajo de 0,70, considerado el nivel adecuado (Nunnally y Berstein, 1994), por lo que podría mostrar problemas de consistencia interna en otras muestras.

En cualquier caso, sería necesario aplicar el CAHV-25 a muestras más amplias, con el fin de comprobar si mantiene las propiedades psicométricas obtenidas en este estudio, así como, incluirlo junto a otras variables psicosociales relacionadas con la violencia escolar, con el fin de estudiar sus posibles aportaciones a los modelos predictivos.

\section{REFERENCIAS}

Ando, M. (2005). Psychosocial Influences on Physical, Verbal, and Indirect Bullying Among Japanese Early Adolescents. Journal of Early Adolescence, 25(3), 268-297.

Andréu, J.M., Peña, M.E. y Graña, J.L. (2001). Agresividad y aceptabilidad de la agresión en jóvenes de ambos sexos. Clínica y Salud, 12(2), 271-284.

Beyers, J.M., Loeber, R., Wikström, P. y Stouthamer-Loeber, M. (2001). What predicts adolescent violence in better-off neighborhooks? Journal of Abnormal Child Psychology, 29, 369-381.

Beynon, J. (1989). A school for men: an ethnographic case study of routine violence in schooling. In J. Archer y K. Browne (Eds.), Human Aggression: Naturalistic Approaches (pp. 122-150). Londres: Routledge and Kegan Paul.

Björkvist, A. (1994). Sex differences in physical, verbal and indirect aggression: A review of recent research. Sex Roles, 30, 177-188.

Cangas, A.J., Gázquez, J.J., Pérez, M.C., Padilla, D. y Miras, F. (2007). Evaluación de la violencia escolar y su afectación personal en una muestra de estudiantes europeos. Psicothema, 19(1), 114-119.

Cerezo, E. y Esteban, M. (1994). El cuestionario BULL. Un procedimiento para la medida de la agresividad entre escolares. Actas del IV Congreso internacional de Evaluación Psicológica. Diputación de Pontevedra.

Costa, M. y Morales, J.M. (1998). ¿Por qué hay niños que cuando jóvenes llegan a comportarse violentamente? Claves para comprender el desarrollo de la violencia. Anuario de psicología jurídica, 8, 163-179.

Defensor del Pueblo (2007). Informe sobre violencia escolar. http://www.defensordelpueblo.es.

Del Barrio, C., Martín, E., Almeida, A. y Barrios, A. (2003). Del Maltrato y otros conceptos relacionados con la agresión entre escolares y estudio psicológico. Infancia y aprendizaje, 26(1), 9-24.

Eagly, A.H. (1987). Sex differences in social behaviour: A social-role interpretation. Hillsdale, NJ: Erlbaum. 
Eagly, A.H. y Chaiken, S. (1998). Attitude structure and function, en D. Gilbert, S. Fiske y G. Lindzey (eds.), Handbook of Social Psychology, $4^{a}$ ed, Vol. 1 (pp. 269-322). Nueva York: McGraw-Hill.

Edward, A.L. (1957). Techniques of attitude scale construction. Nueva York: Appleton-CenturyCrofts.

Etchebarría, I., Apodaca, P., Eceiza, A., Fuentes, M.J. y Ortiz, M.J. (2003). Diferencias de género en emociones y en conducta social en la edad escolar. Infancia y aprendizaje, 26(2), 147-161.

Farrel, A. y Sullivan, T. (2004). Impact of witnessing violence on growth curves for problem behaviors among early adolescents in urban and rural settings. Journal of Community Psychology, 32, 505-525.

Fazio, R.H. (1986). When do attitudes guide behaviour? En R.M. Sorrentino y E.T. Higgins (Eds.), Handbook of Motivation and Emotion: Foundacion of Social Behavior (pp. 204243). Nueva York: Guilford Press.

Fein, S. y Spencer, S.J. (1997). Prejudice as Self-Image Maintenance: Affirming the Self through derogating others. Journal of Personality and Social Psychology, 73(1), 31-44.

García, A. (2001). La violencia en centros educativos de Norteamérica y diferentes países de Europa. Medidas políticas tomadas para la convivencia escolar. Revista Interuniversitaria de Formación del Profesorado, 41, 167-177.

Gázquez, J.J., Cangas, A.J., Pérez, M.C., Padilla, D. y Cano, A. (2007). Percepción de la violencia escolar por parte de los familiares: un estudio comparativo en cuatro países europeos. International Journal of Clinical and Health Psychology, 7(1), 93-105.

Gázquez, J.J., Pérez, M.C., Lucas, F. y Palenzuela, M.M. (2008). Percepción del alumnado universitario sobre el origen de la violencia escolar. European Journal of Education and Psychology, 1(1), 68-80.

Gómez, R., Navarro, M.T. y Ruiz, J.A. (2005a). Construcción de una escala de actitudes hacia la violencia: el CAHV-8. IX Congreso Nacional de Psicología Social. La Coruña.

Gómez, R., Navarro, M.T. y Ruiz, J.A. (2005b). Estudio sobre las actitudes hacia la violencia escolar en adolescentes. En J. Romay y R. García (Eds.), Psicología Social y Problemas Sociales. Vol 5: Psicología Ambiental, Comunitaria y de la Educación (pp. 367-374). Madrid: Biblioteca Nueva.

Hawkins, J.D., Catalana, R.F. y Millar, J.Y. (1992). Risk and protective factors for alcohol and other drug problems in adolescente and early childhood: Implications for substance abuse prevention. Psychological bulletin, 112, 64-105.

Heinemman, P.P. (1972). Mobbing-gruppvald blant barn och vuxna. Stockholm: Naturoch Kultur.

Hoglund, W. (2007). Managing Threat: Do Social-Cognitive Processes Mediate the Link between Peer Victimization and Adjustment Problems in Early Adolescence? Journal of Research on Adolescence, 17(3), 525-540.

Kaiser, H.F. (1960). The application of electronic computers to factor analysis. Educational and Psychological Measurement, 20, 141-151.

Kazdin, A.E. (1988). Tratamiento de la conducta antisocial en la infancia y la adolescencia. Barcelona: Martínez Roca. 
Krauss, S.J. (1995). Attitudes and the prediction of behavior: a metanalysis of the empirical literature. Personality and Social Psychology Bulletin, 21, 58-75.

Lowry, R., Sleet, D., Duncan, C., Powell, K. and Kolbe, L. (1995). Adolescent at Risk for Violence. Educational Psychology, 7, 7-39.

Nunnally, J.C. y Berstein, I.H. (1994). Psychometric theory. Nueva York: McGraw Hill.

Olweus, D. (1973). Personality and aggression. En J.K. Cole y D.D. Jensen (Eds), Nebraska Symposium on Motivation (pp. 261-321). Lincoln University of Nebraska Press: Hemisphere.

Olweus, D. (1978). Aggression in the school: Bullies and whipping boys. Washington D.C: Hemisphere.

Ortega, R. (1994). Violencia interpersonal en los centros educativos de enseñanza secundaria. Un estudio sobre el maltrato y la intimidad entre compañeros. Revista de Educación, 304, 55-67.

Ortega, R. y Mora-Merchán, J.A. (2000). Violencia escolar. Mito o realidad. Sevilla: Mergablum.

Parault, S. (2007). The Social Contexts of Bullying and Victimization. Journal of Early Adolescence, 27(2), 145-174.

Rodríguez, F.J. y Paíno, S. (1994). Violencia y desviación social: bases y análisis para la intervención. Psicothema, 6, 229-244.

Rutter, M., Giller, H. y Hagell, A. (1998). Antisocial behavior by joung people. Cambridge: Cambridge Univestity Press.

Scandroglio, B., Martínez, J.M., Martín, M.J., López, J.S., Martín, A., San José, M.C. y Martín, J.M. (2002). Violencia grupal juvenil: una revisión crítica. Psicothema, 14, 6-15.

Schuette, R.A. y Fazio, R.H. (1995). Attitude accessibility and Motivation as Determinants of Biased Processing: a Test of the MODE model. Personality and Social Psychology Bulletin, 21(7), 704-710.

Taylor, L.D., Davis-Kean, P. and Malanchuk, O. (2007). Self-esteem, academic self-concept, and aggression at school. Aggressive Behavior, 33(2), 130-136.

Tolan, P. (2006). Family violence. Annual Review of Psychology, 57, 557-583

Word, J.J., Cowan, P.A. y Baker, B.L. (2002). Behavior problems and peer rejection in preschool boys and girls. Journal of Genetic Psychology, 163, 72-89. 
Anexo

CAHV-25

(Cuestionario de Actitudes Hacia la Violencia)

A continuación, encontrarás una serie de enunciados sobre lo que los chicos y chicas piensan, sienten o hacen en el centro escolar.

Ten en cuenta que no hay enunciados buenos, ni malos, ni correctos o incorrectos, sólo nos interesa tu opinión sincera.

Trata de responder indicando tu grado de acuerdo con el enunciado utilizando la siguiente escala:

\begin{tabular}{|c|c|c|c|c|c|}
\hline \multicolumn{6}{|l|}{$1=$ Muy en desacuerdo; $2=$ En desacuerdo; $3=$ Indiferente $;=$ De acuerdo $; 5=$ Muy de acuerdo } \\
\hline 01. Me divierte reírme de algunos compañeros & 1 & 2 & 3 & 4 & 5 \\
\hline 02. En la escuela suelo resolver mis problemas usando la fuerza & 1 & 2 & 3 & 4 & 5 \\
\hline 03. Me parece bien meterme con un compañero cuando se lo ha ganado & 1 & 2 & 3 & 4 & 5 \\
\hline 04. Me gusta ver peleas en la escuela & 1 & 2 & 3 & 4 & 5 \\
\hline 05. Me gusta que los demás tengan miedo & 1 & 2 & 3 & 4 & 5 \\
\hline 06. Si me pegara un compañero no le devolvería el golpe $(*)$ & 1 & 2 & 3 & 4 & 5 \\
\hline 07. Pelearse puede ser una forma de divertirse & 1 & 2 & 3 & 4 & 5 \\
\hline 08. Me siento "fuerte" después de insultar a un compañero & 1 & 2 & 3 & 4 & 5 \\
\hline 09. Me gustaría pegar a los que me insultan & 1 & 2 & 3 & 4 & 5 \\
\hline 10. Me gusta poner motes a mis compañeros & 1 & 2 & 3 & 4 & 5 \\
\hline 11. Me siento importante cuando demuestro mi fuerza a los compañeros & 1 & 2 & 3 & 4 & 5 \\
\hline 12. Hay cosas que hay que resolver por la fuerza & 1 & 2 & 3 & 4 & 5 \\
\hline 13. Pelearía para ayudar a un amigo & 1 & 2 & 3 & 4 & 5 \\
\hline 14. Meterse con los profesores es divertido & 1 & 2 & 3 & 4 & 5 \\
\hline 15. Me siento "fuerte" si me meto con mis compañeros & 1 & 2 & 3 & 4 & 5 \\
\hline 16. Pegando los demás te respetan & 1 & 2 & 3 & 4 & 5 \\
\hline 17. La violencia es adecuada para defenderse & 1 & 2 & 3 & 4 & 5 \\
\hline 18. Me gusta hablar de peleas con mis amigos & 1 & 2 & 3 & 4 & 5 \\
\hline 19. Me siento bien agrediendo a los compañeros de clase que me caen mal & 1 & 2 & 3 & 4 & 5 \\
\hline 20. Controlo a mis amigos por medio de la amenaza & 1 & 2 & 3 & 4 & 5 \\
\hline 21. Si me insultara un compañero me defendería atacándole & 1 & 2 & 3 & 4 & 5 \\
\hline 22. Me resulta divertido meterme con algunos compañeros & 1 & 2 & 3 & 4 & 5 \\
\hline 23. A veces me meto con mis compañeros hasta conseguir lo que quiero de ellos & 1 & 2 & 3 & 4 & 5 \\
\hline 24. Me sentiría mal conmigo mismo si para defender a un amigo tengo que pegar a otro chico $(*)$ & 1 & 2 & 3 & 4 & 5 \\
\hline 25. Me gusta meterme con los demás para que se fijen en mí & 1 & 2 & 3 & 4 & 5 \\
\hline $\begin{array}{l}\text { Los ítems } 6 \text { y } 24 \text { puntúan de forma inversa }(1=5 ; 2=4 ; 4=2 ; 5=1) \text {. } \\
\text { Consistencia interna: } 0,90 \text {. Media: } 2,27 \text {. Desviación típica: } 0,59 \\
\text { Factor I: Violencia como forma de diversión (ítems } 1,4,7,10,14,18,22) \text {. Consistencia interna: } 0 \text {, } \\
\text { Factor II: Violencia para mejorar la autoestima (ítems } 5,8,11,15,19) \text { Consistencia interna: } 0,78 \text {. } \\
\text { Factor III: Violencia para manejar los problemas y las relaciones sociales (ítems } 2,12,16,20,2 \\
\text { Desviación típica: } 0,64 \\
\text { Factor IV: Violencia percibida como legítima (ítems } 3,6,9,13,17,21,24) \text { Consistencia interna: } 0\end{array}$ & edia & De & n tí & $\begin{array}{l}\text { ca: } \\
, 66\end{array}$ & 84. \\
\hline
\end{tabular}

Recibido: 28 de agosto de 2008

Recepción Modificaciones: 12 de enero de 2009

Aceptado: 16 de enero de 2009 